\title{
A Fistula between Coronary and Main Pulmonary Arteries with Chest Tightness
}

\author{
Masashi Kawabori ${ }^{*}$, Atsushi Kurata ${ }^{2}$ \\ ${ }^{1}$ Division of Cardiac Surgery, Brigham and Women's Hospital, Harvard Medical School, Boston, MA, USA \\ ${ }^{2}$ Division of Cardiovascular Surgery, Yamato Seiwa Hospital, Yamato, Japan \\ Email: "kawabori.masashi@gmail.com
}

Received 29 October 2015; accepted 11 December 2015; published 14 December 2015

Copyright (c) 2015 by authors and Scientific Research Publishing Inc.

This work is licensed under the Creative Commons Attribution International License (CC BY).

http://creativecommons.org/licenses/by/4.0/

(c) (i) Open Access

\section{Abstract}

A 64-year-old woman presented to our facility with recurrent chest tightness. Angiography showed a dilated $(10 \mathrm{~mm})$ aneurysmal tortuous coronary artery fistula (CAF) to the main pulmonary arterial trunk occupying a large area of the anterior surface of the aortic root. Left and right heart catheterization showed high left-to-right shunt flow. Fissurectomy and coronary artery bypass grafting were performed. The patient had no postoperative complications and became asymptomatic. The outcome of our case shows that CAF should be a differential diagnosis of recurrent symptomatic cardiac ischemia. Intervention is indicated if symptoms or secondary complications develop.

\section{Keywords}

Congenital, Coronary Aneurysm, Coronary Fistula, Angina

\section{Introduction}

Coronary artery fistulas (CAFs) are uncommon vascular communications between a coronary artery and a great vessel around the heart or one of the cardiac chambers. CAFs are the most frequent congenital anomaly of the coronary arteries [1]. Most CAFs are small and remain asymptomatic. However, the coronary artery steal phenomenon may occur, causing myocardial ischemia of the segment perfused by the coronary artery distal to the CAF and dilation of the proximal artery to compensate for the flow. Symptomatic patients commonly present with heart failure secondary to volume overload resulting from left-to-right shunting, ischemia due to coronary

"Corresponding author. 
steal, arrhythmia, fistula rupture or thrombosis, and infective endocarditis; in these cases, interventions are indicated [2]. Surgical treatment is previously the only option, but transcatheter interventions are emerging as alternatives in patients with suitable anatomy and/or without other complex cardiac lesions [3]. We present herein a case of chest pain caused by a CAF that is successfully treated with surgery.

\section{Case Report}

A 64-year-old woman with a history of well-controlled non-familial hypercholesterolemia presented with recurrent chest tightness on effort. The patient had no previous history of coronary disease or smoking. The only medication she was taking was atorvastatin.

At admission, an electrocardiogram showed no abnormal findings. Transthoracic echocardiography showed good ejection fraction with no valvular disorders and elevated right ventricular systolic pressure of $41.2 \mathrm{mmHg}$. Nonetheless, computedtomography was performed to screen for coronary artery disease. Angiography revealed a dilated aneurysmal tortuous CAF occupying a large area of the anterior surface of the aortic root (Figures 1(a)-(c)). The CAF originated from the proximal left anterior descending (LAD) coronary artery through the fistula; the exact number of fistulas was unidentifiable due to the tortuous morphology. The left main coronary artery (LM) and CAF were dilated to $10 \mathrm{~mm}$, and it was defined as a "large" fistula and coronary aneurysm [4]. A small LAD appeared distal to the fissure. No thrombus was seen in the CAF. Left and right heart catheters were performed to evaluate coronary flow and steal (Online Video 1). Coronary angiography also revealed a dilated LM, and contrast examination showed a tortuous dilated fistula draining from the proximal LAD into the main trunk of the PA. Further hemodynamic study revealed a pulmonary-to-systemic flow ratio of 1.54:1, left left-to-right shunt ratio of $32.4 \%, 11.7 \% \mathrm{O}_{2}$ step-up between the right ventricle and main PA, and PA pressure elevated to $42.5 \mathrm{mmHg}$. Based on these findings, the patient was diagnosed with symptomatic coronary artery ischemia resulting from the coronary artery steal phenomenon from high flow shunting from left to right with coronary aneurysmal change. Surgery was considered to eliminate the recurrent symptomatic coronary flow steal of the fistula and because of the risk of rupture of the aneurysmal dilatation of the coronary fistula. In the surgical view, the dilated fistula (Figure 2) exhibited a noticeable thrill. Fissurectomy and patch closures to both entries of the fistula were performed. The coronary artery was patched with the saphenous vein, and the pulmonary artery was patched with a bovine pericardial patch (Edward Bovine Pericardial Patch, Edwards Life Sciences, CA, USA). Coronary artery bypass grafting surgery was performed by connecting the end of the left internal mammary artery to the LAD.

The patient responded well to the surgical intervention. Postoperative coronary artery computed tomography showed no residual left-to-right shunt, and echocardiography showed good left ventricular function. Right ventricle systolic pressure decreased to $37.2 \mathrm{mmHg}$. The patient was discharged two weeks after surgery. She has had no complications and has remained asymptomatic after 2 years of follow-up.

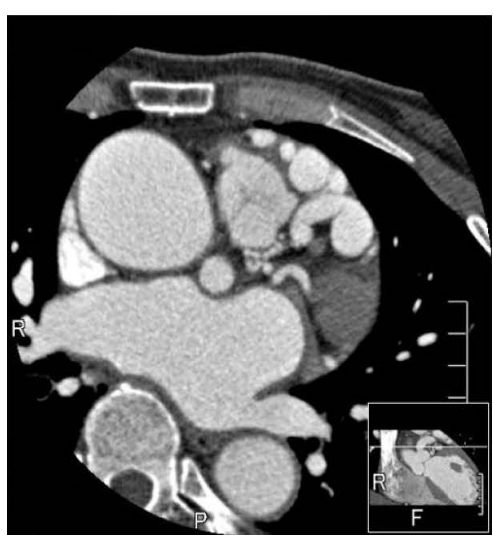

(a)

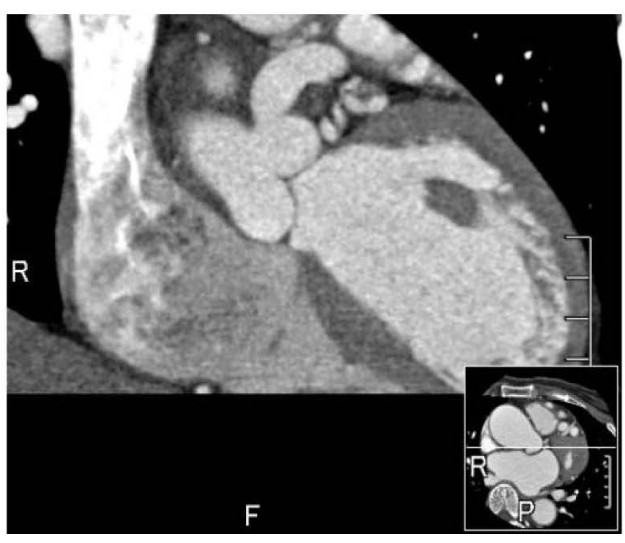

(b)

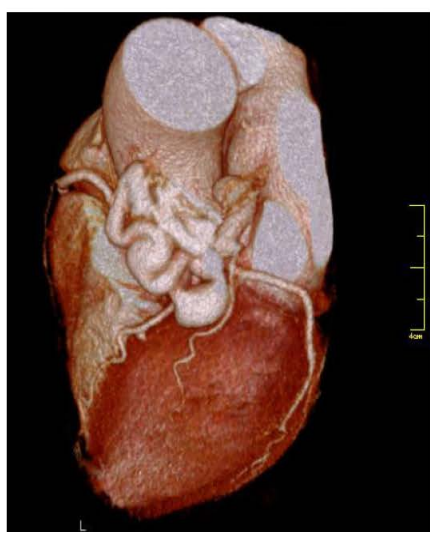

(c)

Figure 1. (a) Coronary artery fistula reconstruction. Computed tomography image showing multiple dilated fistulas; (b) Computed tomography angiography (CTA) showing aneurysmal change of the left main coronary artery; (c) 3D CTA showing an aspect of a tortuous coronary aneurysmal coronary shunt. 


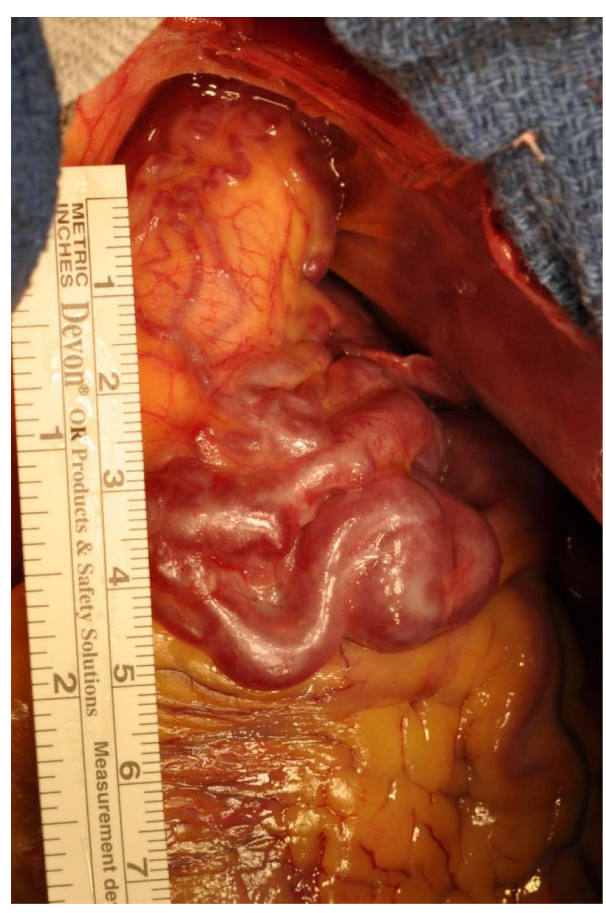

Figure 2. Image showing an intraoperative aspect of the large tortuous fistula.

\section{Discussion}

According to management strategy, interventions are indicated for CAF with any clinical symptoms. However, although management for asymptomatic fistulas remains controversial, it is prudent to have an invasive strategy for asymptomatic fistulas when the pulmonary-to-systemic flow ratio exceeds 1.5:1 or when aneurysms form, as this can lead to mural thrombosis, distal embolization, rupture, and dissection [5]. There are also indications for asymptomatic high-flow shunting to prevent occurrence of complications such as bacterial endocarditis and pulmonary hypertension [6]. Surgical intervention was previously the only method for treating CAF with excellent long-term efficacy and safety [7]. Catheter-based interventional techniques have become viable options for CAF closure since 1983, when the first percutaneous closure was reported, though long-term outcomes after fistula occlusion with catheter devices remain unknown. Catheter interventions are acceptable if the anatomical features are not complicated. Selection of occluding devices also depends on anatomical findings. In complicated anatomical cases such as extreme tortuosity of fistulous tract or aneurysm formation, we recommend surgical closure of the fistula.

\section{Conclusion}

As our case shows, though CAF is a rare cardiac anomaly, it should be a differential diagnosis of recurrent symptomatic cardiac ischemia. Intervention for CAF is indicated if the patients are symptomatic or if other secondary complications develop.

\section{Disclosure Statement}

The authors declare no conflict of interest.

\section{References}

[1] Kwon, S.H., Oh, J.H., Hwang, S.J., Kim, S.J., Kim, M.G., et al. (2010) Bilateral Coronary Artery Fistulae to Main Pulmonary Discovered Incidentally upon Multidetector-Row Computed Tomography. Texas Heart Institute Journal, 37, 127-128.

[2] Guedes, M.A., Riso Ade, A., BarberoMarcial, M. and Stolf, N.A. (2009) Off-Pump Correction of Coronary Fistula: 
The Single Suture Technique Approach. Revista Brasileira de Cirurgia Cardiovascular, 24, 581-583. http://dx.doi.org/10.1590/S0102-76382009000500023

[3] Jama, A., Barsoum, M., Bjarnason, H., Holmes, D.R. and Rihal, C.S. (2011) Percutaneous Closure of Congenital Coronary Artery Fistulae. JACC: Cardiovascular Interventions, 4, 814-821. http://dx.doi.org/10.1016/j.jcin.2011.03.014

[4] Szalat, A., Durst, R., Cohen, A. and Lotan, C. (2005) Use of Polytetrafluoroethylene-Covered Stent For Treatment of Coronary Artery Aneurysm. Catheterization and Cardiovascular Interventions, 66, 203-218. http://dx.doi.org/10.1002/ccd.20448

[5] Angelini, P. (2002) Coronary Artery Anomalies Current Clinical Issues: Definitions, Classification, Incidence, Clinical Relevance, and Treatment Guidelines. Texas Heart Institute Journal, 29, 271-278.

[6] McMahon, C.J., Nihill, M.R., Kovalchin, J.P., Mullins, C.E. and Grifka, R.G. (2001) Coronary Artery Fistula. Management and Intermediate Term Outcome after Transcatheter Coil Occlusion. Texas Heart Institute Journal, 28, 21-25.

[7] Kamiya, H., Yasuda, T., Nagamine, H., Sakakibara, N., Nishida, S., et al. (2002) Surgical Treatment of Congenital Coronary Artery Fistulas: 27 Years' Experience and a Review of the Literature. Journal of Cardiac Surgery, 17, 173177. http://dx.doi.org/10.1111/j.1540-8191.2002.tb01195.x

\section{Supplementary Online Video 1}

Angiography demonstrating a dilated tortuous fistula left main coronary artery aneurysm and a fistula to the main pulmonary artery.

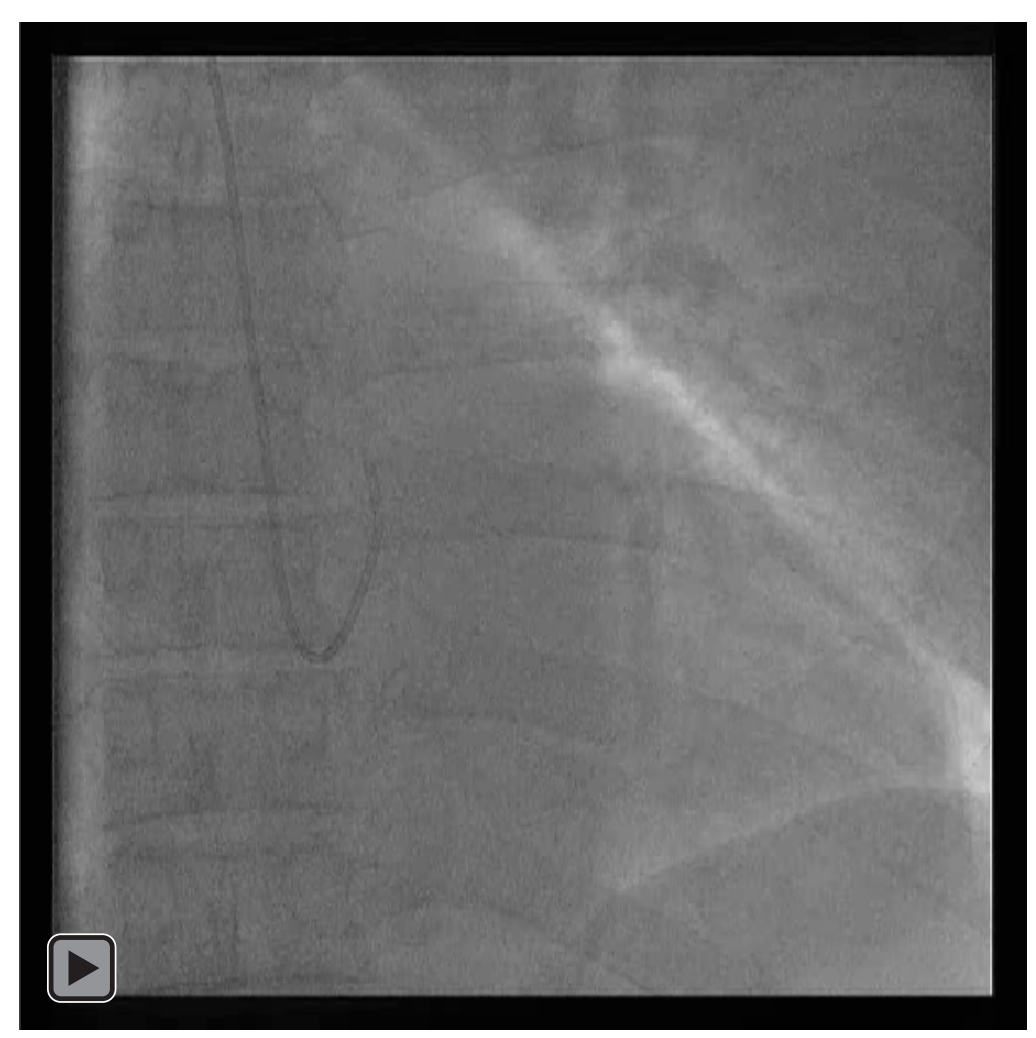

